\title{
Effect of Surface Modes on Coupling to Fast Waves in the LHRF
}

\author{
R. I. Pinsker and P. L. Colestock \\ Plasma Physics Laboratory, Princeton University \\ Princeton, NJ 08544
}

\begin{abstract}
ABSTRACT

The effect of surface modes of propagation on coupling to fast waves in the LHRF is studied theoretically and experimentally. The previously reported 'updown' poloidal phasing asymmetry for coupling to a uniform plasma is shown to be due to the properties of a mode which carries energy along the plasnaconducting wall interface. Comparison of the theory with coupling experiments performed on the PLT tokamak with a phased array of twelve dielectric-loaded waveguides at $800 \mathrm{MHz}$ shows that the observed dependence of the net reflection coefficient on toroidal phase angle can be explained only if the surface wave is taken into account.
\end{abstract}




\section{INTRODUCTION}

In recent years, efficient non-inductive current drive using the slow wave in the lower hybrid range of frequencies (LHRF) $\Omega_{i} \ll \omega \ll \Omega_{e}$ bas been demonstrated in many tokamak experiments[1]. However, the extrapolation of this current drive technique to a tokamak reactor is not straightforward, because of the observed current drive density limit for a fixed frequency and the problem of wave accessibility to the core of a large, hot plasma at high frequencies. For this reason, several recent experiments have been carried out to investigate current drive with the other propagating plasma wave in the IHRF: the fast wave[2-7].

It was shown theoretically in Refs, $[8,9]$ that efficient coupling to the fast wave with a phased array of open-ended waveguides is much more difficult than coupling to the slow mode. In this paper, we show that under some circumstances, much of the incident of power from a fast wave antenal in the LHRF is coupled not to the desired propagating fast wave, but to a non-penetrating surface mode which carries energy away from the antenna along the plasma boundary. This surface mode does not correspond to the waveguide-like modes involving spectral energy in $1<n_{\|}^{2}<n_{\| \text {crit }}^{2}$ discussed by Brambilla[10], nor to the similar mode involving fast waves with $n_{\|}^{2}<1$ propagating in the low density edge region studied by Theilhaber and Bers[9]. Both of the latter modes require a density or magnetic field gradient, while the surface wave discussed here can exist at the boundary of a plasma with $\nabla n_{e}, \nabla B_{0}=0$ everywhere in the plasma. The surface mode here discussed is related to the surface wave in the ICRF discussed by Messiaen, et al.[11], while th= mode of Theilhaber and Bers is clearly analogous to the 'coaxial mode' in the ICRF also discussed in Ref. [11]. It is shown that the 'up-down' poloidal phasing asymmetry for coupling to a uniform plasma reported in Ref. [12] is due to the properties of the surface mode.

We then compare the theory with the results of coupling experiments performed on the PLT tokamak wich a $3 \times 4$ phased array of dielectric-loaded waveguides at $800 \mathrm{MHz}[6]$. The observed dependence of the net reflection coef- 
ficent on toroidal phase angle can be explained only if the surface wave is taken into account. With this caveat, general agreement between the coupling theory and the experimental results is demonstrated.

\section{THEORY OF THE SURFACE MODE}

We consider only the phased waveguide array coupler, for which the theory bas been exhaustively developed $[9,10,12-16]$, and we shall use the notation of, and frequently refer to, Ref. [12]. However, our conclusions concerning the surface mode and its importance apply to other coupler designs; in particular, the surface mode may also be important in experiments employing loop antennas $[4,5]$ or other types of couplers[7].

Since it was shown in Ref. [12] that many of the qualitative features of the coupling physics are determined only by the plasma parameters near the waveguide openings, we first consider the situation in which the plasma adjacent to the coupler is uniform. (We later will relax this restriction.) For the case with $n_{e}=1.1 \times 10^{12} \mathrm{~cm}^{-3}, B_{0}=20 \mathrm{kG}, f=800 \mathrm{MHz}$, Fig. 9 of [12] shows[17] that when the phasing is such that most of the spectral power is concentrated near $n_{y}=-1$ (but not $n_{y}=+1$ ), $n_{z}=0$, very low net reflection coefficients are found. At this value of density and magnetic field, neither siow ner fast waves can propagate in the plasma with this $\left(n_{y}, n_{x}\right)$, as the slow lower bybrid wave must have $n_{z}^{2}>1$ to propagate above $n_{e}=n_{c}=8 \times 10^{9} \mathrm{~cm}^{-3}$, while the fast wave with $n_{x}^{2}<1$ can propagate only at densities below $n_{\mathrm{e}}$ lectoff $\simeq 3.47 \times$ $10^{-2} f B\left(I-\pi n_{z}^{2}\right)$, that is, below $n_{e} \simeq 5.5 \times 10^{11} \mathrm{~cm}^{-3}$ in this case[10,9]. Therefore, with these parameters the power may propagate away from the antenna only along the plasma-conducting wall interface.

Since the mode that carries most of the power away from the antenna evidently is most important for $n_{z} \approx 0$, we shall temporarily consider the case where $n_{z}=0, n_{y} \neq 0$, corresponding to a poloidal array of infinitely wide waveguides. This is the opposite linit from that considered by Brambilla[10] and Theilhaber and Bers[9], where it was assumed that $n_{y}=0, n_{z} \neq 0$. In 
the algorithm used in this work, all of the information about the plasma is embedded in the admittance matrix $Y\left(n_{y}, n_{z}\right)$ which connects the transverse components of the wave electric and magnetic fields at $x=0^{+}: \mathbf{B}_{T}\left(\pi_{y}, n_{z}, z=\right.$ $\left.0^{+}\right)=Y\left(n_{y}, n_{x}\right) \cdot E_{T}\left(n_{y}, n_{x}, x=0^{+}\right)$, in which $\mathbf{E}_{T}=\mathbf{E} \cdot(I-\hat{x} \hat{x})$ and similarly for $\mathbf{B}_{T}$. For the uniform case under consideration here, analytic expressions for $Y$ may be simply derived, and are given in $[16,18]$ for slow and fast wave excitation, respectively. For our present purposes, we note that Eq. (2) of [12] gives the denominator of each of the four components of $Y_{i j}$ as

$$
\begin{aligned}
E_{y}^{\text {fast }}\left(x=0^{+}, n_{y}, n_{x}\right) E_{z}^{\text {dlow }}\left(x=0^{+}, n_{y}, n_{x}\right) \\
-E_{y}^{\text {slove }}\left(x=0^{+}, n_{y}, n_{x}\right) E_{z}^{\text {fast }}\left(x=0^{+}, n_{y}, n_{x}\right) .
\end{aligned}
$$

Also, the approximation $E_{z}^{\text {fast }}=0$, which yields the expression of Ref. [18] for the fast wave admittance of a uniform plasma

$$
Y_{21} \approx \frac{B_{z}}{E_{y}}=\frac{n_{y}^{\text {fart }}\left(S-n_{z}^{2}\right)-i D n_{y}}{S-n_{x}^{2}-n_{y}^{2}},
$$

in which $n_{z}^{\text {fast }}=n_{z}^{f a s t}\left(n_{y}, n_{z}\right)$ is the index of refraction for the fast wave in the $x$-direction, becomes exact for $n_{z}=0$. In this case, the fast and slow waves completely decouple, and are conventionally[19] referred to as the extraordinary $(X)$ and ordinary $(O)$ modes, respectively. Furthermore, if $n_{z}=0$, the dispersion relation for the fast $(X)$ mode reduces to $n_{z}^{f a s t}=\sqrt{\frac{R L}{S}-n_{Y}^{2}}$. The appropriate branch of the square root is determined by causality: for $R L / S>r_{y}^{2}$, the condition that the mode carry enersy only in the positive $x$-direction (away from the antenna) implies that $n_{\mathrm{z}}>0$ (forward wave), while for $R L / S<n_{y}^{2}$, the wave amplitude must decay for $x>0$, so that $n_{x}=+i \sqrt{n_{y}^{2}-\frac{R L}{S}}$. For the LHRF, $S \leqslant 1, D \approx \omega_{p e}^{2} /\left(\omega \Omega_{e}\right)$, and since $R L=S^{2}-D^{2}$ identically, for $\omega<\omega_{p e}^{2} / \Omega_{e}, \pi_{2}^{f a s t}$ is pure positive imaginary for any value of $\pi_{y}$.

Substitution of this $\mathrm{X}$-mode dispersion relation into Eq. 2 with $n_{x}=0$ reveals a simple pole at $n_{y}=-\sqrt{S}$, but not at $n_{y}=+\sqrt{S}$. Now, it is easily shown that, in general, if $\operatorname{Re}\left(Y_{i j}\right)=0$, the $x$-component of the time-averaged Poynting flux varishes, i. e. the real part of the admittance represents power flow away from the coupling structure. With $\omega<\omega_{\text {pe }}^{2} / \Omega_{e}$ and $S>0$ (below the lower hybrid 
resonance density), $Y_{21}$ is purely imaginary, but the causal resolution of the pole, guaranteed by the inclusion of an imaginary part to $n_{y}$ with the proper sign[12], yields a spike in the real part of $Y_{21}$ at the pole. The width of the spike is proportional to $\operatorname{Im}\left(n_{y}\right)$ and its height is inversely proportional to $\operatorname{Im}\left(n_{y}\right)$, so that the residue of the pole (the area beneath the spike) is independent of the size of the imaginary part introduced.

The pole thus implies power flow away from the antenna. Since the plasma does not support a propagating mode at $\left(n_{y}, n_{x}\right)=(-\sqrt{S}, 0)$, the Poynting vector must lie in the $x=0$ plane as $\operatorname{Im}\left(n_{y}\right) \rightarrow 0$. One can visualize the introduction of the small imaginary part to $\left(n_{y}, n_{z}\right)$ as a slight distortion of the $x=0^{+}$surface so that power flowing along $x=0$ eventually passes through this surface at large $z$ or $y$. No matter how small $\operatorname{Im}\left(n_{y}, n_{z}\right)$ is taken to be, all of the energy leaving the antenrta is now properly accounted for, whether it flows into the plasma or along the surface.

This property of the algorithm was pointed out by Brambilla[10]; he showed that the power trapped between the slow and fast wave cutofis and the modeconversion point in an inhomogeneous plasza is represented by poles in the admittance matrix elements, and be resolved the poles in a way equivalent to the method adopied here. In an earier report [15], Brambilla considered the case in which the plasma is replaced by a vacuum layer of finite thickness, bounded by a perfectly conducting sheet parallel to the $x=0$ plane. This stripline can carry power only in the direction parallel to the walls, so that the real part of the admittance is composed of a series of spikes corresponding to the eigenmodes of the stripline. For fast wave launching into a plasma with a low density at $x=0^{+}$, the waves with $\left|n_{1}\right|<1$ can form similar eigenmodes between $x=0$ and the fast wave cutoff, and Theilhaber and Bers[9] demonstrated that these eigenmodes imply poles in the admittance appropriate to their problem.

However, none of these waveguide-like modes can occur when the plasma is taken to be uniform for $\approx>0$. But under some circumstances, a true surface mode[20,21] can exist at the interface between two media with different dielectric or ferromagnetic properties. Suck a mode is evanescent in both media in the 
direction normal to the interface but carries energy along the interface. Perhaps the best-known example of this phenomenon in plasma physics is the TrivelpieceGould mode[22] that exists at the interface between an unmagnetized plasma and a vacuum or dielectric. Another surface mode exists at the interface between two magnetized cold plasmas or between a magnetized plasma and a vacuum or dielectric $[23,24]$ with the static magnetic field parallel to the interface. A similar surface wave at the interface between a cold magnetized plasma and a perfectly conducting sheet was described by Seshadri $[25,26]$. The boundary conditions at a perfectly conducting sheet imply that the electric field of the mode must be norwal to the interface; it results from a surface charge on the plasma. In the absence of a surface charge of magnetic monopoles, the magnetic field in the plasma must lie in the plane $x=0^{+}$, so that the Poynting vector of the mode also lies in the plane, as required for a surface wave. Next, we explicitly show that such a mode can be constructed using only plasma modes decaying away from the interface; simple reflection at $x>0$ mimics the surface mode, but is clearly not a causal solution in the situation under consideration.

The superposition of the two causal solutions in the uniform cold magnetized plasma permit us to satisfy the two boundary conditions

$$
\begin{aligned}
& E_{y}\left(x=0^{+}\right)=E_{y}^{\text {sow }}+E_{y}^{f a \Delta t}=0 \\
& E_{z}\left(x=0^{+}\right)=E_{z}^{\text {low }}+E_{z}^{\text {fast }}=R_{\text {low }} E_{y}^{\text {sow }}+R_{\text {fast }} E_{y}^{\text {fast }}=0
\end{aligned}
$$

where the ratio $R \equiv E_{x} / E_{y}$ for each of the two modes is determined by the plasma parameters, $n_{y}$, and $n_{z}$. These two equations are satisied if $R_{\text {slow }}=$ $R_{\text {fost }}$, which is also exactly the condition that the denominator of the $Y$ matrix elements (Eq. 1) vanish. Thus we see that the existence of a pole in the $Y$ matrix elements for the uniform plasma implies the propagation of the surface mode along the plasma-conducting sheet interface. It is also now clear that no surface wave exists in this situation as $B_{0} \rightarrow 0$ : the two cold plasma modes become degenerate in this limit and $R_{\text {slow }}=R_{\text {fast }}$ trivially.

As shown above, the case of $n_{x}=0$ so that the surface wave propagates normal to $B_{0}$ can be treated simply, because the two cold plasma modes com- 
pletely decouple. Then we may satisfy the condition $E_{z}=0$ by exciting only the X-mode. For the X-mode[19], $E_{y} / E_{x}=\left(n_{y}^{2}-S\right) /\left(n_{y} n_{x}-i D\right)$, or

$$
\frac{E_{y}}{E_{x}}=\frac{i\left(S-n_{y}^{2}\right)}{n_{y} \sqrt{n_{y}^{2}+\frac{D^{2}-S^{3}}{S}}-D}
$$

where, again, we have chosen the causal root of $n_{z}$. Now, $E_{y}=0$ is satisfied by $n_{y}= \pm \sqrt{S}$, but if we substitute $n_{y}=+\sqrt{S}$ back into $\mathrm{Eq}$. 3 , we see that both numerator and denominator vanish for this choice. Therefore, the only proper root is

$$
n_{y}=-\sqrt{S}
$$

and we once again arrive at the dispersion relation for the surface wave with $n_{z}=0$.

The surface wave exists only for densities below the lower hybrid resonance density, at which $S=0$; for densities much lower than this limiting value, such as is characteristic of edge plasma in a tokamak for the LHRF, the surface wave dispersion approaches that of a vacuum wave propagating poloidally along the plasma surface $\left(n_{z}=0, n_{y}=1\right)$. For the parameters of Ref. [12], Fig. 9, a surface mode propagates with $n_{z}=0$ at $n_{y}=-0.995$.

It is natural to attempt to construct an O-mode surface wave for $n_{z}=0$, as this mode satisfies $E_{y}=0$ identically. But an O-mode decaying in the positive $x$-direction cannot satisfy $E_{x}=0$ nontrivially, so that an array of waveguides oriented to excite the slow wave cannot couple directly to a surface wave. This explains why for slow wave cases the code described in Ref. [12] agrees with simpler codes that cannot model this phenomenon. Another way to see that there is no $\mathrm{O}$-mode surface wave is to observe that $\mathrm{O}$-mode dispersion is independent of magnetic field, so that a hypothetical 0 -mode surface wave would exist as $B_{0} \rightarrow 0$. But it has been shown above that no surface mode exists on a perfectly conducting sheet as $B_{0} \rightarrow 0$.

We may generalize the surface mode dispersion relation for $n_{z} \neq 0$, as was done for the similar surface mode at a unagnetized plasma-vacuum interface in 
Ref. [23], by setting $R_{\text {slaw }}=R_{\text {fast }}$, or

$$
\begin{aligned}
& \frac{\left.n_{z}\right|_{\text {slow }}\left(S-\left.n_{z}^{2}\right|_{\text {slow }}-n_{z}^{2}\right)-n_{y}\left(\left.n_{y} n_{z}\right|_{\text {elow }}+i D\right)}{\left(\left.n_{y} n_{x}\right|_{\text {olow }}+i D\right)\left(P-\left.n_{z}^{2}\right|_{\text {olow }}-n_{y}^{2}\right)-\left.n_{y} n_{z}^{2} n_{x}\right|_{\text {olow }}}= \\
& \frac{\left.n_{x}\right|_{\text {fast }}\left(S-\left.n_{z}^{2}\right|_{\text {fast }}-n_{z}^{2}\right)-n_{y}\left(\left.n_{y} n_{x}\right|_{\text {fast }}+i D\right)}{\left(\left.n_{y} n_{x}\right|_{\text {fast }}+i D\right)\left(P-\left.n_{x}^{2}\right|_{\text {fost }}-n_{y}^{2}\right)-\left.n_{y} n_{z}^{2} n_{z}\right|_{\text {fast }}}
\end{aligned}
$$

in which $\left.n_{z}\right|_{\text {alow }},\left.n_{z}\right|_{\text {fast }}$ are the solutions of the cold plasma dispersion relation with positive imaginary part. This equation is sufficiently complicated that solutions must be found numerically. Such a solution for the parameters of Ref. [12], Fig. 9 is shown in Fig. 1; a surface wave exists for $n_{y}, n_{z}$ near the unit circle for $\theta \$ 30^{\circ}$, where $\tan \theta \equiv \pi_{y} / n_{z}$.

We can demonstrate that the surface mode dominates the coupling behavior shown in Ref. [12], Fig. 9 and in the inhomogeneous case shown in Fig. 8, op cit. by setting $\operatorname{Re}\left(\mathrm{Y}_{i j}\right)=0$ for $\left|n_{z}\right|<\left.n_{z}\right|_{\text {crit }}=1.16$ (minimum accessible $n_{z}$ for $n_{\mathrm{e}}=1.1 \times 10^{12} \mathrm{~cm}^{-3}, B_{0}=20 \mathrm{kG}$ ) and recomputing them. This does not permit the surface wave to carry energy away from the antenna. The contours of constant reflection for the two cases are shown in Figs. 2 and 3. Comparison of these plots with the corresponding plots in Ref. [12] in which the surface wave is taken into account shows that essentially all of the power leaving the antenna for phasings near $0^{\circ}$ toroidal, $-90^{\circ}$ poloidal is coupled to the surface wave. No significant up-down asymmetry remains in the uniform plasma case, and the optimum toroidal phase angle is now such that most of the spectral energy is between the accessibility $\left(\left|n_{z}\right|>1.16\right)$ and cutoff $\left(\left|n_{z}\right|<1.73\right)$ limits. The remaining up-down asymonetry in the inhomogeneous case is attributable to the effect of the density gradient discussed in Refs. [8] and [27].

We next describe a simple calculation which makes these numerical results plausible, by analytically demonstrating the good impedance match which can be obtained between a waveguide array and the surface mode. We take the case of a single, infinitely wide, dielectric-filled waveguide as an idealization of an infinite (in the toroidal direction) array of waveguides excited in phase, and assume that only the reflected fundamental mode of the waveguide is important. By carrying out the matching procedure of Brambilla[13], we obtain the 
following expression for the reflection coefficient $\rho$ :

$$
\frac{1-\rho}{1+\rho}=\Lambda \equiv \frac{i k_{0} a}{2 \pi \sqrt{\epsilon}} \int_{C} d n_{y} \frac{S \sqrt{n_{y}^{2}+\frac{D^{2}}{S}-S}-n_{y} D}{S-n_{y}^{2}}\left[\frac{\sin ^{2}\left(\frac{k_{0} a}{2} n_{y}\right)}{\left(\frac{k_{0} a}{2} n_{y}\right)^{2}}\right],
$$

in which $a$ is the height of the waveguide, $k_{0} \equiv \omega / c$ and $\epsilon$ is the dielectric constant of the material filling the wayeguide, and the integral is to be computed over the contour shown in Ref. [12], Fig. 2b. The power reflection coefficient is given by

$$
|\rho|^{2}=\frac{[1-\operatorname{Re}(\Lambda)]^{2}+[\operatorname{Im}(\Lambda)]^{2}}{[1+\operatorname{Re}(\Lambda)]^{2}+[\operatorname{Im}(\Lambda)]^{2}}
$$

and we see explicitly that if $D^{2}>S^{2}$, the only power transmitted is due to the surface mode at $n_{y}=-\sqrt{S}$. If we assume that the contribution from the pole is much larget than the the principal value, we find $\Lambda \simeq \frac{\alpha}{\sqrt{6}} \frac{D}{S}\left(\frac{\sin \alpha}{\alpha}\right)^{2}$, where $\alpha \equiv \frac{1}{2} k_{0} a \sqrt{S}$. If we evaluate this for the plasma parameters of Ref. [12], Fig. 9, and take $a=5.4 \mathrm{~cm}, \epsilon=8.0$, we obtain $|\rho|^{2} \approx 0.30$. If $a=0.5 \mathrm{~cm}$ instead, we estimate $|\rho|^{2} \approx 0.89$. Using the code described in Ref. [12] to model a single row of four waveguides $8.6 \mathrm{~cm}$ wide by 5.4 or $0.5 \mathrm{~cm}$ high, separated by $0.67 \mathrm{~cm}$ in the toroidal direction, radiating into a uniform plasma with the parameters of Ref. [12], Fig. 9, we obtain $|\rho|^{2}=0.33$ and 0.92 , respectively, when the four guides are excited in phase. These are the power reflection coefficients for the inner pair of guides; the guides on the ends are calculated to have a somewhat highe: $|\rho|^{2}$. Considering the simplicity of the estimate, the agreement between the numerical result and the analytic estimate is excellent. We may thus conclude that the low reflection coefficients found in Ref. [12] result from the good impedance match between the wareguide and the surface mode. Unfortunately, this implies that the regime of low reflection coefficionts discussed in Ref. [12] is of no use in fast wave current drive experiments, since almost none of the launched power penetrates the surface of the plasma. This is in agreement with the direct computation of the power spectrum that reaches the plasma core reported in Ref. [12].

The effect of a vacuum layer between the plasma edge ar.d the conducting wall on coupling and propagation of the surface wave may be assessed 
by computing the phase change of the wave in propagating across the gap: $\Delta \phi=i k_{0} \int_{0}^{x_{x}} n_{x} d x$, in which $n_{y}^{2}=1-\pi_{y}^{2}-n_{x}^{2}$. For the X-mode surface wave with $n_{x}=0,|\Delta \phi|=k_{0} x_{p}|1-S|^{1 / 2} \approx x_{p}\left(\omega_{p i} / c\right)\left|1-\left(\omega / \omega_{g m g}\right)^{2}\right|^{1 / 2}$, in which the geometric mean gyrofrequency $\omega_{g m g} \equiv \sqrt{\Omega_{i} \Omega_{e}}$. Under typical conditions in the LHRF, this phase change is negligible compared to unity; for the example considered above, the gap would have to be about $60 \mathrm{~cm}$ to produce $|\Delta \phi| \sim 1$. Generally, a gap large enough to significantly affect the surface mode would also preclude efficient coupling to propagating fast waves $\left(n_{s}^{2}>1\right)$, due to the long tunneling distance. In the experiment discussed in this paper, the gap between the plasma edge and the conducting vacuum vessel was less than $10 \mathrm{~cm}$, and the other parameters were such that the effect of the gap on the surface mode was negligible.

A substantial vacuum region between the plasma and the conducting wall permits the propagation of the 'coaxial mode'[11], which is also the limit of the mode discussed by Theilhaber and Bers[9] in which the linear density profile is replaced by a vacuum region and a step. This mode is not a true surface wave; rather, it corresponds to a strip line with the plasma and the conducting sheet forming the two walls.

The relationship between the coaxial mode and the surface mode considared in this paper can be quantified by calculating the dispersion relation for the case where the plasma density is zero for a distance $0<x<x_{p}$ in front of the conducting sheet, then uniform for $x>x_{p}$. Matching tangential electric and magnetic field components at the plasma/vacuum baundary, and tangential electric fields at the conducting sheet, we obtain the dispersion relation for $n_{x}=0$;

$$
\sqrt{1-n_{y}^{2}} \tan \left(k_{0} x_{p} \sqrt{1-n_{y}^{2}}\right)=\frac{n_{y}^{2}-S}{S \sqrt{n_{y}^{2}-R L / S}-D n_{y}} .
$$

If the gap is small, $k_{0} x_{p} \ll 1$, and the dispersion relation for the surface mode is given approximately by $n_{y}=-\sqrt{S}-k_{0} x_{p} D(1-S)$. As $k_{0} x_{p} \sim \pi$, new pairs of solutions appear, which correspond to the coaxial modes mentioned above. It can be shown that the lowest order pair of coaxial mades occurs when the 
gap is between $\lambda_{0} / 2$ and $\lambda_{0} / 2$ wide, where the vacuum wavelength $\lambda_{0}=\mathrm{c} / \mathrm{f}$. For the case of Ref. [12], Fig. 9, except now permitting a vacuum gap, we find the lowest order coaxial modes appear at $z_{p} \geq 15.3 \mathrm{~cm}\left(z_{p}=0.41 \lambda_{0}\right)$.

In situations where the vacuum gap is large enough to permit both the surface mode and the coaxial modes to propagate, and the antenna spectrum contains energy in the ranges of $\left(n_{y}, n_{x}\right)$ where these modes can propagate, both types of modes will be excited. We may evaluate the partition of energy between the coaxial modes and the surface mode by comparing the residue at the poles of $Y_{21}$ which correspond to those modes. Again considering the only analytically tractable case, where $n_{2}=0$, we find that the admittance is given by

$$
Y_{21}=i \frac{S \sqrt{n_{y}^{2}-R L / S}-D n_{p}-\left(S-n_{y}^{2}\right) \tan \left(k_{0} x_{p} \sqrt{1-n_{y}^{2}}\right)}{S-n_{y}^{2}+\sqrt{1-n_{y}^{2}} \tan \left(k_{0} x_{p} \sqrt{1-n_{y}^{2}}\right)\left(S \sqrt{n_{y}^{2}-R L / S}-D n_{y}\right)} .
$$

The residue can be easily evaluated at a pole, where the denominator of Eqn. 8 vanishes (Eqn. 7 is satisfied), by evaluating the quotient of the numerator of Eqn. 8 and the derivative with respect to $n_{y}$ of the denominator at the pole. In Fig. 4, we plot the residue of $F_{21}$ at the poles as a funcion of the size of the vacuum gap, normalized to the residue with $x_{p}=0$, which is equal to $D$. The plasma parameters are again those of Ref. [12], Fig. 9 . For $x_{p}>15.3 \mathrm{~cm}$, the coaxial modes (one propagating in the positive $y$ direction, the other in the negative $y$ direction) are more important power sinks than the surface mode.

Finally, we consider the effect of a sinooth density gradient on the surface mode. In the foregoing, we showed that for a uniform plasma, the residue at the pole of $Y_{21}\left(n_{y}, n_{x}\right)$ that representa the surface mode is equal to $D$ for $D^{2}>S^{2} \simeq$ 1. We therefore might expect that the residue of $Y_{21}$ at the pole with a density ramp replacing the density step would be smaller, since $D \simeq \omega_{p e}^{2} /\left(\omega \Omega_{e}\right) \sim n_{e}$. However, because $\sqrt{S}$ is very close to unity throughout the edge region in the LHRF, the value of $n_{y}$ at which the pole occurs would not be expected to be strongly affected by finite $d n_{e} / d x$. These qualit ative expectations are borne out by numerical calculation of $Y_{21}\left(n_{y}, n_{z}=0\right)$ with a linear density ramp. The location of the pole and the residue of $Y_{21}$ there were computed as a function 
of the density gradieat scale leagth $a$, with $n_{e}(2\}=\left(1.1 \times 10^{12} \mathrm{~cm}^{-9}\right)(z / a)$ for $0<x<a, n_{e}=1.1 \times 10^{12} \mathrm{~cm}^{-9}$ for $\approx>a$. The mngnetic field was $20 \mathrm{kG}$, and the jon mass $m_{i}=2 m_{p}$. As mentioned above, for $a=0$, the resicine is $D \approx 2.0$; we plot the resioue of $Y_{21}$ normalized to this value in Fig. 5. Though the level of the coupling between a given antemna and the surface mode depends on the density gradient in front of the anternb, the gross properties of the mode are unchanged by finite $d n_{e} / d x$.

\section{COMPARISDN OF THEORY AND EXPERIMENT}

The experiments were performed usiug a $? \times 4$ array of dielectric $(\epsilon=2.8)$ filled waveguides; each waveguide $F E ; .25 \mathrm{~cm}$ wide (in the toroidal direction) and $5.08 \mathrm{~cm}$ high (in the poloidal direction). The guides were mounted in a steel frame which maintained a distance of $0.7 \mathrm{~cm}$ between the guides in the toroidal direction and $2.9 \mathrm{~cm}$ in the polnidal direction[28]. The array was mounted in an cutside midplane port on the Princeton Large Torus (PLT)[29], a circular cross-section tokamak with $x=132 \mathrm{~cm}, a=40 \mathrm{~cm}$. The limiters were placed at minor radii in the range $i=3 \$$ to $a=43 \mathrm{~cm}$ during these experiments; a pair of Braphite limiters were fixed at either side of the waveguide array at $a=43 \mathrm{~cm}$. The plasma current and line-averaged density were varied over the ranges 200 $-400 \mathrm{kA}$ and $1 \times 10^{12} \mathrm{~cm}^{-3}-3 \times 10^{13} \mathrm{~cm}^{-3}$, respectively. Deuterium gas was used. Rf power at $800 \mathrm{MHz}$ was suppled by the same set of klystrons as had been used for lower hybrid slow wave current drive experiments on PLT[30,31]; the If system was described in detail in Ref. [32]. Further information concerning the waveguide array, the tokamak, the rf system, and diagnostic techniques is given in Ref. [33].

The experimental results on waveguide array-plasma c suplin: al wa be simmarized as follows. With fixed plasma conditions, the net reflection coefficient $R$ as a function of toroidal phase angle $\Delta \phi_{T}$ always had a minimuxn at $\Delta \phi_{T}=0^{\circ}$, and a broad maximure at $\Delta \phi_{T}$ uear $130^{\circ}$, as is shown in Figs. 6 and 7 a. As the line-averaged density $\bar{n}_{e}$ was raised with $\Delta \phi_{T}$ fxed, $R$ dropped substantially 
(Figs. ? and 8a). Both of these dependences are in sharp cantrast to the behavior observed in experiments with slow wave arrays (Fig. 6b, for example). As has been shown in Refs. [34-36] and others, the observed behavior of slow wave arrays is in good agreement with the well-developed coupling theory.

To compare results from the coupling theory to the experiment, one must bave information on the density profile in the neighborhood of the coupler. Langmuir probe data[37] provided a measurement of the density profile in the range $a=42-45 \mathrm{~cm}$, though the absolute value of the edge density was not calibrated, and these measurements were not performed under conditions precisely identical to those under which the waveguide coupling data was taken. Absolute values for the edge density and its dependence on $\bar{n}_{e}$ were obtained by fitting the results of $2.45 \mathrm{GHz}$ lower hybrid slow wave coupling measurements to the theory of Ref. [16]; this procedure bas been shown to give values of edge density in agreement with detailed probe measurements[38].

For simulation of the pbase scan shown in Fig. 6 , in which $\vec{x}_{e}=1.5 \times$ $10^{1.3} \mathrm{~cm}^{-3}, I_{p}=400 \mathrm{kA}, B_{T}=31.3 \mathrm{kG}$, and $r_{l i m}=40 \mathrm{~cm}$, we take $n_{\mathrm{e}}(r=$ $43 \mathrm{~cm})=1.3 \times 10^{11} \mathrm{~cm}^{-3}$, and $\left(\nabla n_{e}\right)$ ledge $=4 \times 10^{11} \mathrm{~cm}^{-4}$. This linear profile is assumed to extend to the limiter radius, and the profile inside the limiter is taken to be parabolic. The predicted contours of constant net reflection coefficient[12] for this case are shown in Fig. 9. As discussed in Sec. 2, the effect of the surface wave is to predict a strong up-down poloidal phasing asymmetry, which was not observed. Furthermore, the dependence of $\mathrm{R}$ on $\Delta \phi_{T}$ at a fixed poloidal phasizs of $0^{\circ}$ (the conditions under which the data of Fig. 6 was taken) is unlike what was seen in the experiment, with $R$ predicted have a me:ximum at $\Delta \phi_{T}=0^{\circ}$. Rather, the observed dependence was more like that predicted for near-optimal peloidal phasing, so that the surface wave with $n_{z}=0, n_{y} \simeq-1$ is strongly excited with $\Delta \phi_{T} \simeq 0^{\circ}$. A simple error in poloidal phase calibration is ruled out by the fact that we found no significantly better coupling at any other poloidal phasing.

If the poloidal phasing were for some reason ineffective, so that the array asted like three independent $1 \times 4$ arrays, then the width of the launched poloidal 
spectrum would increase by a factor of $\sim 4$. The surface wave loading would be strong only for toroidal phasings near $0^{\circ}$, and the up-down asymmetry of that mode mould not be observable from reflection coefficient data alone. The toroidal phase, dependence of the coupling under these circumstances can be computed simply by using a $1 \times 4$ array in the code. The dependence of $\mathrm{R}$ on $\Delta \phi_{T}$ thus calculated is shown in Fig. 10, where it is compared with the analogous curve obtained from the full $3 \times 4$ case at $\Delta \phi_{P}=0^{\circ}$. The qualitative behavior observed (Fig. 6a) is elearly much closer to that predicted by assuming that the rows of the array sre effectively decoupled.

There is, in fact, a good reason to expect that the poloidal phasing might be considerably less effective than the toroidal phasing in our experimental configuration, where the spaces between the rowa were somewhat more than half the height of the guides, while the toroidal spacing was $<10 \%$ of the width of the guides. The theory described in [12], like all theories of the Brambilla type, assiumes that a perfectly conducting sheet fills all the spaces between the waveguide openings and the remainder of the $x=0$ plane. The surface currents that flow on the conducting sheet in the idealized model permit discontinuity in the transverse magnetic field at $\approx=0$; this effect is quite important[15]. Bench tests with single[39] and double waveguides[40] have shown important differences in the fields in the plane of the waveguide openings between situations with and without a ground plane. As pointed out by Greene[39], currents flowing on the outer surface of the waveguides can be substantial; these currents would be expected to produce sharp peaks in the electric field at the edges of the guides, and consequently to spread the launched spectrum to bigher $\left|n_{\|}\right|$than predicted by the idealized theory. In our experiment, arc tracks which were observed on the top and bottom sturfaces of the waveguides are evidence that strong fringing fields existed in the kz:ps between the rows of guides.

We conclude that a reasonable model for the coupling is to assume that the $3 \times 4$ array acted as though it were composed of three independent $1 \times 4$ arrays. The exeited $n_{y}$-spectrum is thus about four times as broad as it would have been in the true $3 \times 4$ case, so that though the spectrum is peaked at $n_{y}=0$, 
the surface wave at $n_{y} \simeq-1, n_{x}=0$ is still strongly excited for toroidal phase angles $\$ 90^{\circ}$. To explain the minimum in $R$ at $\Delta \phi_{T}=0^{\circ}$, we must invoke the surface wave - Fig. 10a also shows th: $R\left(\Delta \phi_{T}\right)$ predicted for the $4 \times 1$ array with $\operatorname{Re}\left(Y_{i j}\right)$ set equal to zero for $n_{x}^{2}<1$, so that the surfacs wave is not allowed to carry energy away from the coupler. Comparison of these curves with the experimental data (Fig. 6a) shows that the $\mathrm{X}$-mode surface wave appears to dominate the loading for phase angles near $\Delta \phi_{T}=0^{\circ}$.

We model the two-guide density scan of Fig. 7 a by assuming proportionality between $\bar{n}_{e}$ and $\left(\nabla n_{e}\right) l_{e d g c}$, as suggested by the edge density measurements, that $\left.n_{\mathrm{e}}\right|_{\text {edge }}$ is fixed at $1.3 \times 10^{21} \mathrm{~cm}^{-3}$, and that $n_{\mathrm{e} 0}=1.5 \bar{n}_{\mathrm{e}}$, as is appropriate for a parabolic profile. We compute the "scattering matrices" for a $1 \times 4$ array, but then treat the outer pair of guides as passive when computing $R$ - in the experiment the outer guides were terminated. The results of this computation are shown in Fig. 7b. Given that the magnitude of $R$ was not absolutely caijbrated in Fig. 7a, the qualitative agreement between the model results and the experiment is rather good.

The same seattering matrices were used to predict the reflection coefficient as a function of $\bar{n}_{e}$ with a fixed toraidal phase angle of $180^{\circ}$, for comparison with the data shown in Fig. 8a. In this case, all four guides were powered. The observed reduction in $R$ as $\bar{n}_{e}$ is raised is accurately reproduced by the theory but the magnitude of $\mathrm{A}$ found in the experiment was about 0.2 lower than the theoretical value for all densities. A similar offset is seen comparing the theory and experiment for the phase scan at fixed density (Figs. 6a and 1Ca). The simplest explanation for this discrepancy is that some fraction $f$ ' of the power is lost in the coaxial lines connecting the directional couplers and the waveguide array. Since this loss is squared for the reflected power, the true reflection coefficient would be $(1-f)^{-1}$ times the value measured at the directional coupler. The experimental data of Fig. 8a is replotted in Fig. 8b with an assumed loss of $f=25 \%(1.25 \mathrm{~dB})$, and compared with the theory. This $f$ has been chosen to give the best fit to the data.

Though the agreement between the theory and the experiment is quite good, 
assuming this fairly large loss in the lines, in view of the differences between the experimental configuration and the idealized model, such good agreement must be regarded as somewhat fortuitous. In particular, the gaps between the guides are not modelled properly, poloidal curvature effects are not included in the model, and the density profile is not known well enough for a precise comparison of theory and experiment. The fast wave coupling region extends much farther into the plasma than does the region that determines slow wave coupling. Stevens, et al.[16] showed that for most experimental situations, the slow wave coupling efficiency depends only on a single parameter: the density at the face of the coupler, while fast wave coupling relies on tunneling through the outer few centimeters of the profile and is thus dependent on the density profile in a much larger region. The effect of density fluctuations on coupling is an unsolved problem, but one might expect that when the cutoff layer is located near the limiter radius, where fluctuation levels are bighest, any such effect would be maximized. These fluctuations may have a decisive effect on the nature of the power that actually penetrates the plasma[41,42], so it is perhaps reasonable to expect some effect on the coupling.

We conclude that given the greater sensitivity of fast wave coupling to factors not well characterized, either theoretically or experimentally, the level of quantitative agreement between coupling theory and experiment seen in the slow wave case is not to be expected. However, the extent of the qualitative agreement that we do find shows that the basic coupling physics of the dielectric-loaded waveguide array fast wave coupler is reasonably well understood. In particular, the antenna could not have launched a large fraction of the incident power cirectly into the slow wave branch without qualitatively changing the dependence of $R$ vs. $\bar{n}_{c}$ and vs. $\Delta \phi_{\mathrm{T}}$. Carrying out the spectral analysis described in [12] for the case where $\Delta \phi_{T}=180^{\circ}, \bar{n}_{e}=5 \times 10^{12} \mathrm{~cm}^{-3}$, we find that $<5 \%$ of the power that escapes the coupling region is on the slow wave branch. 


\section{SUMMARY AND CONCLUSIONS}

We have made a detailed comparison between coupling measurements using a $3 \times 4$ dielectric-loaded waveguide array and a complete linear coupling model including both slow and fast modes in the lower-hybrid range of frequencies. We have shown both analytically and numerically that the poloidally asymmetric coupling characteristics described in previous theoretical work are largely due to the excitation of surface modes. Moreover, these waves can dominate the coupling properties over a wide range of plasma edge conditions and toroidal phase angles. The experimental results indicate a coupling dependence on toroidal phase angle the we can explain only by invo'ing the presence of such surface waves. Good qualitative agreement between theory and experiment was obtained; although certain nonideal conditions in the experiment, such as the presence of sizable gaps in the ground plane between adjacent waveguides and losses in the transmission lines, give rise to a plausible absolute discepancy between our idealized model and the experimental results. We coaclude that these surface waves are likely to piay an important role in attempts[43] to couple to the fast wave in this frequency range.

\section{ACKNOWLEDGMENTS}

The auth.rs would like to thank G. J. Greene for the Langmuir probe data he so kindly permitted us to use, and A. Bers and J. E. Stevens fre many useful discussions.

This work was supported by U.S. Department of Energy Covitraet No. DEAC02-76CHO3073. 


\section{References}

[1] FISCH, N. J., Rev. Mod. Phys. 59(1987) 175.

[2] GOREE, J., ONO, M., COLESTOCK, P., HORTON, R., McNEILL, D., PARK, H., Phys. Rev. Lett. 55 (1985) 1669.

[3] McWILLIAMS, R., PLATT, R. C., Phys. Rev. Lett. 56 (1986) 835.

[4] OHFUBO, K., HAMADA, Y., OGAWA, Y., et al., Phys. Rev. Leti. 56 (1986) 2040.

[5] UESUGI, Y., et al., in Applications of Radio-Frequency Power to Plasmas (Proc. 7th Top. Conf., Kissimmee, FL, 1997), American Institute of Physies, New York (1987) 179.

[6] PINSKER, R. I., COLESTOCK, P. L., BERNABEI, S., CAVALLO, A., GREENE, G. J., KAITA, R., STEVENS, J.E., in Applications of Radio-Frequency Power to Plasmas (Proc. Tth Top. Conf., Kissimmee, FL, 1987), American Institute of Physics, New York (1987) 175.

[7] COLBORN, J. A., Current-Drive on the Versator II Tokamak with a Slotted-Wavegruide Fast-Wave Coupler, M. S. dissertation, Massachusetts Institute of Technology (1987), MIT Plasma Fusion Center Report No. PFC/RR-87-20.

[8] BERGER, R. L., PERKINS, F. W., TROYON, F., "Coupling of Slow and Fast Waves to Plasmas Near the Lower Hybrid Frequency", Priaceton Plasma Physics Lab. Rep. PPPL-13E6 (Aug. 1977).

[9] THEILHABER, K., BERS, A., Nucl. Fusion 20 (1980) 547.

[10] BRAMBILLA, M., Nucl. Fusion 19 (1979) 1343.

[11] MESSIAEN, A. M., KOCH, R., BHATNAGAR, V. P., VANDENPLAS, P. E., WEYNANTS, R. R., in Heating in Toroidal Plasmas (Proc. $\{$ th Int. Symp., Rome, 1984), int. School of Plasma Phys. and ENEA, Perugia, Italy (1984), Vol. I, 315. 
[12] PINSKER, R. I., DUVALL, R. E., FORTGANG, C. M., COLESTOCK, P. L., Nucl. Fusion 28 (1986) 941.

[13] BRAMBIL:LA, M., Nucl. Fusion 16 (1976) 47.

[14] BRAMBILLA, M., "Review of the Grill Theory", Association Euratom-CEA Rep. ETJR-CEA-FC 890 (April 1977).

[15] BRAMBILLA, M., "Radiation from Waveguide Arrays", Association Euratom-CEA Rep. EUR-CEA-FC 906 (July 1977).

[16] STEVENS, J., ONO, M., HORTON, R., WILSON, J. R., Nucl. Fusion 21 (1981) 1259.

[17] We wish to point out a typographical error in [12]; the captions . appropriate to Figs. 8 and 9 were inadvertantly switched. Thus, in the present work, 'Fig. 8 of [12]' reiers to the plot labeled Fig. 9 there, and vice-versa.

[18] FORTGANG, C. M., COLESTOCK, P. L., HWANG. D. Q., in Course and Workshop on Applications of RF Waves to Takamak Plasmas (Int. School of Plasma Phys., Vurenna, 1985), International School of Plasma Physies, Perugia, Italy (1985) 408.

[19] STIX, T. H., The Theory of Plasma. Waves, McGraw-Hill, New York (1962).

[20] ALLIS, W. H., BUCHSBAUM, S. J., BERS, A., Waves in Anisotmpic Plasmas, MIT Press, Cambridge, MA (1963).

[21] PORTIS, A. M., Electromagnetic Fields: Sounces and Media, John Wiley and Sons, New York (1978), 498ff.

[22] TRIVELPIECE, A. W., GOULD, R. W., J. Appl. Phys. 20 (1959) 1784.

[23] KOTSARENKO, N. Ya., SINKEVICH, E. L., FEDORCHENKO, A. M., Sov. Phys.-Tech. Phys. 16 (1971) 640. 
[24] ELGIN, J. N., J. Plasma Physics 14 (1975) 255.

[25] SESHADRI, S. R., IRE Trans, on Microwave Theory and Techniques, MTT-10 (1962) 573.

[26] SESHADRI, S. R., IEEE Trans, on Microwave Theory and Techniques, MTT-11 (1963) 238.

[27] McWILLIAMS, R., MOK, Y., Fusion Technol. 7 (1985) 283.

[28] The $3 \times 4$ dielectric-loaded waveguide array was constructed by Grumman Aerospace Corporation, Bethpege New York.

[29] GROVE, D., et al, in Plasma Physics and Controlled Nuclear Fusion Research, 1976 (Proc. 6th Int. Conf. Berchtesgader, West Germany, 1976), Vol.1, IAEA, Vienna (1977) 21.

[30] BERNABEI, S., DAUGHNEY, C., EFTHIMION, P., HOOKE, W., et al., Phys. Rev. Lett. 49 (1982) 1255.

[31] JOBES, F. C., et al., Phys. Rev. Lett. 52 (1984) 1005.

[32] SCHNABL, F. J., MARTIN, A. R., NEWMAN, W. G., in Engineering Problems of Fusion Research (Prac. 9th Symp., Chicago, 1981), IEEE, New York (1981) Vol. 1, 851.

[33] PINSKER, R. I., Fast Wave Curvent Drive Experiments on the Princeton Large Torus, Ph. D. dissertation, Princeton University (1988).

[34] BERNABEI, S., HEALD, M. A., HOOKE, W. M., MOTLEY, R. W., PAOLONI, F. J., BRAMBLLA, M., GETTY, W. D., Nucl. Fusion 17 (1977) 929.

[35] Gormezano, C., MOREaU, D., Plasma Phys. and Controlled Fusion $26(1984) 553$.

[36] OHKUBO, K., MATSUmotO, K., Jap. J. Appl. Phys. 26 (1987) 142.

[37] The Langmuir probe data was provided by G. J. Greene. 
[38] STEVENS, J. E., et al., Nucl. Fusion 28 (1988) 217.

[39] GREENE, G. J., et al., in Applications of Radio-Frequency Power to Plasmas (Proc. 7th Top. Conf., Kissimmee, FL, 1987) American Institute of Physics, New York (1987) 322.

[40] MOTLEY, R. W., GREENE, G. J., in RF Plasma Heating (Proc. 6th Top. Conf., Callaway Gardens, GA, 1985), American Institute of Physics, New York (1985) 170.

[41] ANDREWS, P. L., Fhys. Rev. Lett 54 (1985) 2022.

[42] ANDREWS, P. L., BHADRA, D. K., Nucl. Fusion 26 (1986) 827.

[43] PINSKER, R. I., et al., manuscript in preparation. 


\section{Figures}

Fig. 1. The solution of Eq. 5, plotted as $r \equiv \sqrt{n_{y}^{2}+n_{z}^{2}}$ as a function of $\theta \equiv$ $n_{y} / n_{z}$. The plot for $90^{\circ}<\theta<270^{\circ}$ is obtained by reficiting the plot around $\theta=90^{\circ}$.

Fig. 2. Contours cf power reflection coefficient for uniform plasma with $n_{e}=$ $1.1 \times 10^{22} \mathrm{~cm}^{-3}, B_{0}=20 \mathrm{kG}$, with surface waves not allowed to carry energy, to be compared with Ref. [12], Fig. 9.

Fig. 3. Contours of power reflection coefficient for the 'standard' fast wave array case of Ref. [12], Fig. 8 , with the surface waves removed.

Fig. 4. The residue of the admittance $Y_{21}$ at the poles, with $n_{x}=0$, with a vacuum gap and a deuterium plasma with density $1.1 \times 10^{22} \mathrm{~cm}^{-3}$ for $x>x_{p} ; B_{0}=20 \mathrm{kG}$.

Fig. 5. The residue of the admittance $Y_{21}$ at the surface wave pole, with $n_{2}=0$, as a function of the density gradient scale length $a$, normalized to the residue with $a=0$. The density at the end of the ramp is $1.1 \times 10^{12} \mathrm{~cm}^{-3} ; B_{0}=20$ $k \mathrm{G}$.

Fig. 6. Comparison of observed toroidal phase dependence of net reflection coefficient with slow and fast wave $800 \mathrm{MHz}$ couplers. (a) Net reflection coefficient for $3 \times 4$ array vs. toroidal phase angle. $B_{T}=31.3 \mathrm{kG}, \bar{n}_{e}=$ $1.5 \times 10^{13} \mathrm{~cm}^{-3}$, forward power $190 \mathrm{~kW}$. (b) Similat curve obtained with $1 \times 6$ slow wave coupler[38]. $B_{T}=28 \mathrm{kG}, \bar{r}_{e}=1.85 \times 10^{13} \mathrm{~cm}^{-3}$, forward power $12 \mathrm{~kW}$.

Fig. 7. Relative net power reflection for 2-waveguide subset of the $3 \times 4$ array vs. toroidal phase angle at six different line-averaged densities. $P_{\text {rf }} \sim 1$ $\mathrm{kW}$. The curves are fits of the form $a+b \cos \phi$. These curves are a relative measure of the plasma loading, and were calibrated by assuming that the minimum in $\mathrm{R}$ for the vacuum occurs at $\Delta \phi_{T}=0^{\circ}$, and taking the maximum reflection in the vacuum to be $\mathrm{R}=1$. [The dielectric filling makes 
the waveguides a poor impedance match to the vacuum, so that the pair of guides with $\Delta \phi_{T}=180^{\circ}$ are predicted to have a reflection coefficient of 0.93 in a vacuum, as shown in (b).] Having established the calibration with the vacuum curve, the phase scan was repeated at each value of $\bar{n}_{e}$.

$$
\begin{array}{lll}
0=\text { vacuum } & \times=0.5 \times 10^{13} \mathrm{~cm}^{-3} & \Delta=1.1 \times 10^{19} \mathrm{~cm}^{-3} \\
\square=1.5 \times 10^{13} \mathrm{~cm}^{-3} & \bullet=2.0 \times 10^{13} \mathrm{~cm}^{-3} & \diamond=2.6 \times 10^{13} \mathrm{~cm}^{-3}
\end{array}
$$

(b) $R\left(\Delta \phi_{T}\right)$ predicted for two-waveguide density scan. Line-average densities of $\bar{n}_{\mathrm{e}}=$ vacuum, $5 \times 10^{12} \mathrm{~cm}^{-3}, 1 \times 1 \mathrm{c}^{13} \mathrm{~cm}^{-3}, 1.5 \times 10^{13} \mathrm{~cm}^{-3}, 2 \times 10^{13} \mathrm{~cm}^{-3}$, and $2.5 \times 10^{23} \mathrm{~cm}^{-3}$.

Fig. 8. (a) Observed net power reflection for $3 \times 4$ fast wave array as a function of $\bar{n}_{\mathrm{e}}$ with $\Delta \phi_{T}=180^{\circ}, I_{p}=500 \mathrm{kA}, B_{T}=31.3 \mathrm{kG}$. (b) R( $\left.\bar{n}_{\mathrm{e}}\right)$ predicted for density scan witb $\Delta \phi_{T}=180^{\circ}$, and compared to experimental data, where a loss in the coaxial lines of $25 \%$ has been assurned.

Fig. 9. Contours of power refeetion coeffeient for modeling of phase scan of Fig. 6 .

Fig. 10 Combaring the predictions of $\mathrm{R}\left(\angle \phi_{T}\right)$ for three models. (a) $1 \times 4$ array, with surface waves allowed (solid) and forbidden (dashed). (b) $3 \times 4$ array with $\Delta \phi_{P}=0^{\circ}$. 


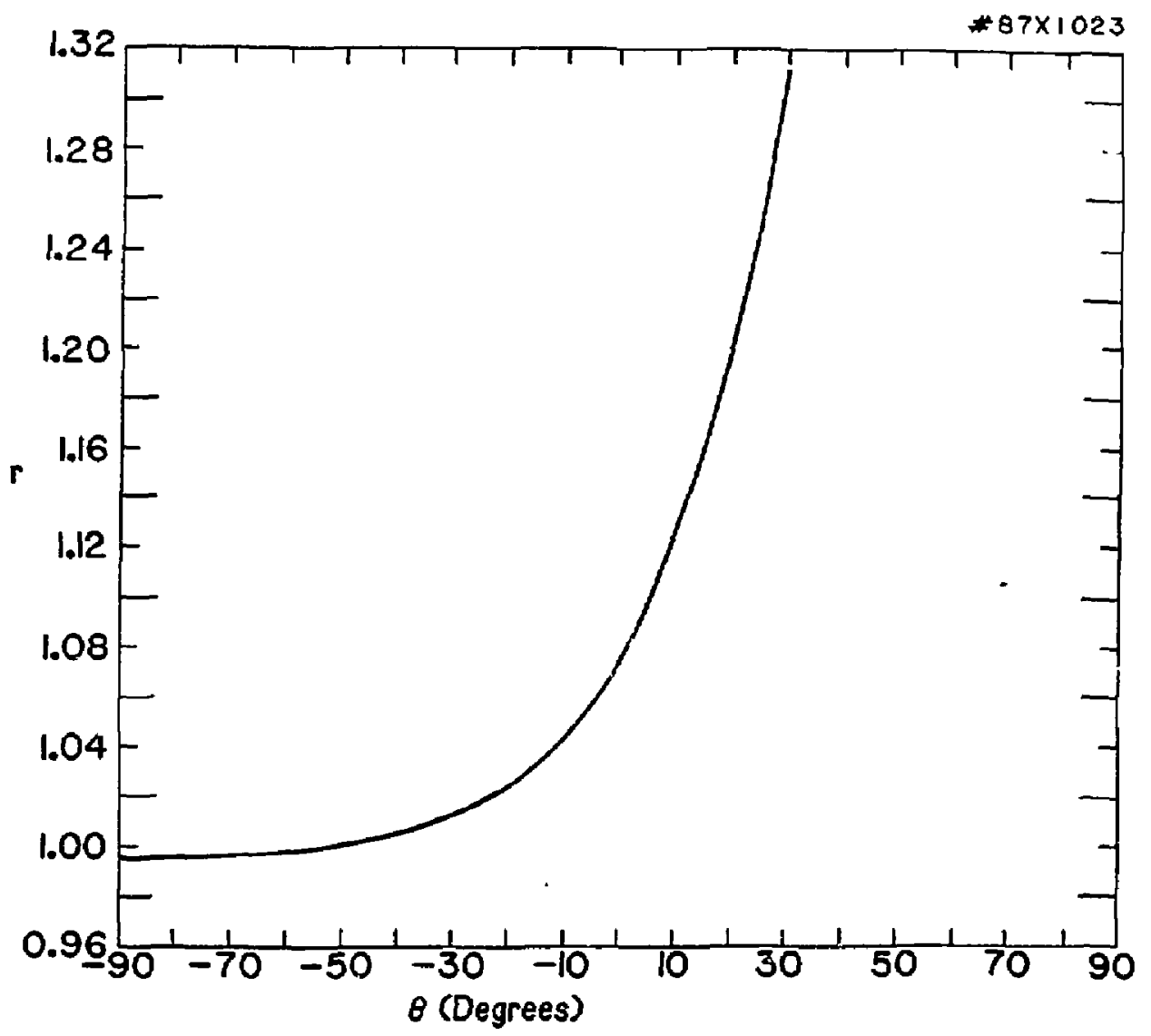




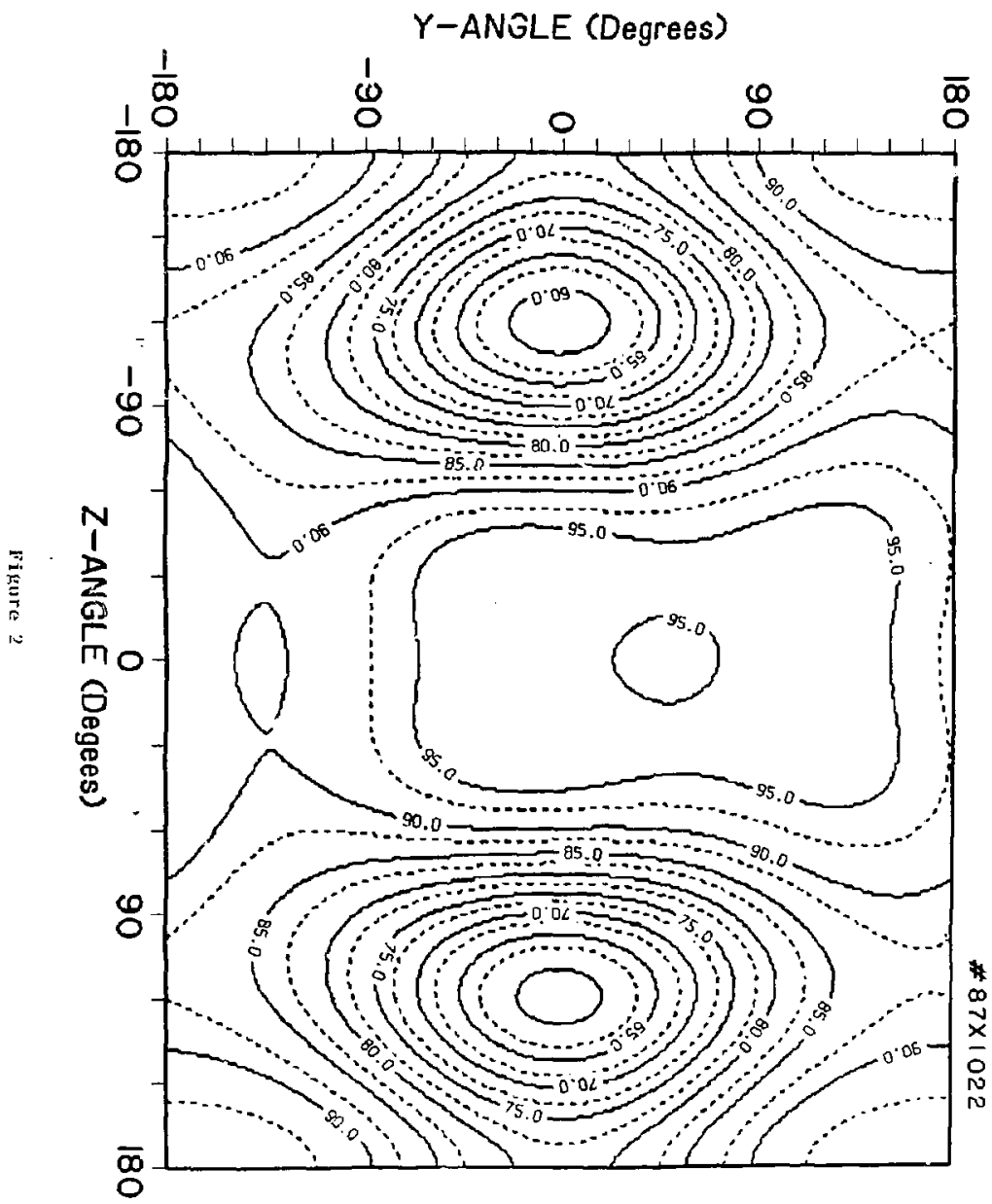




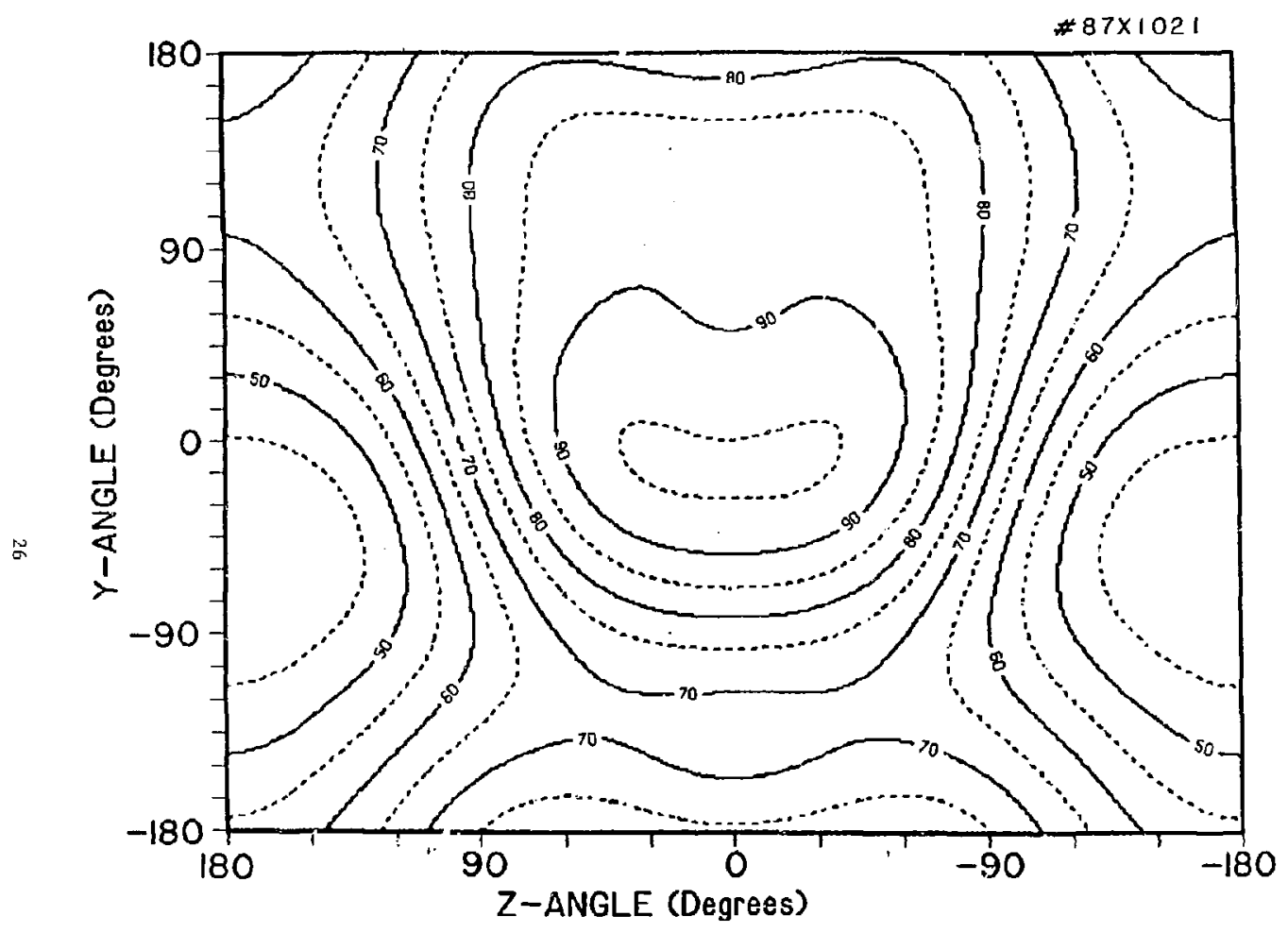

Figures ; 
PPPL \#90×0523

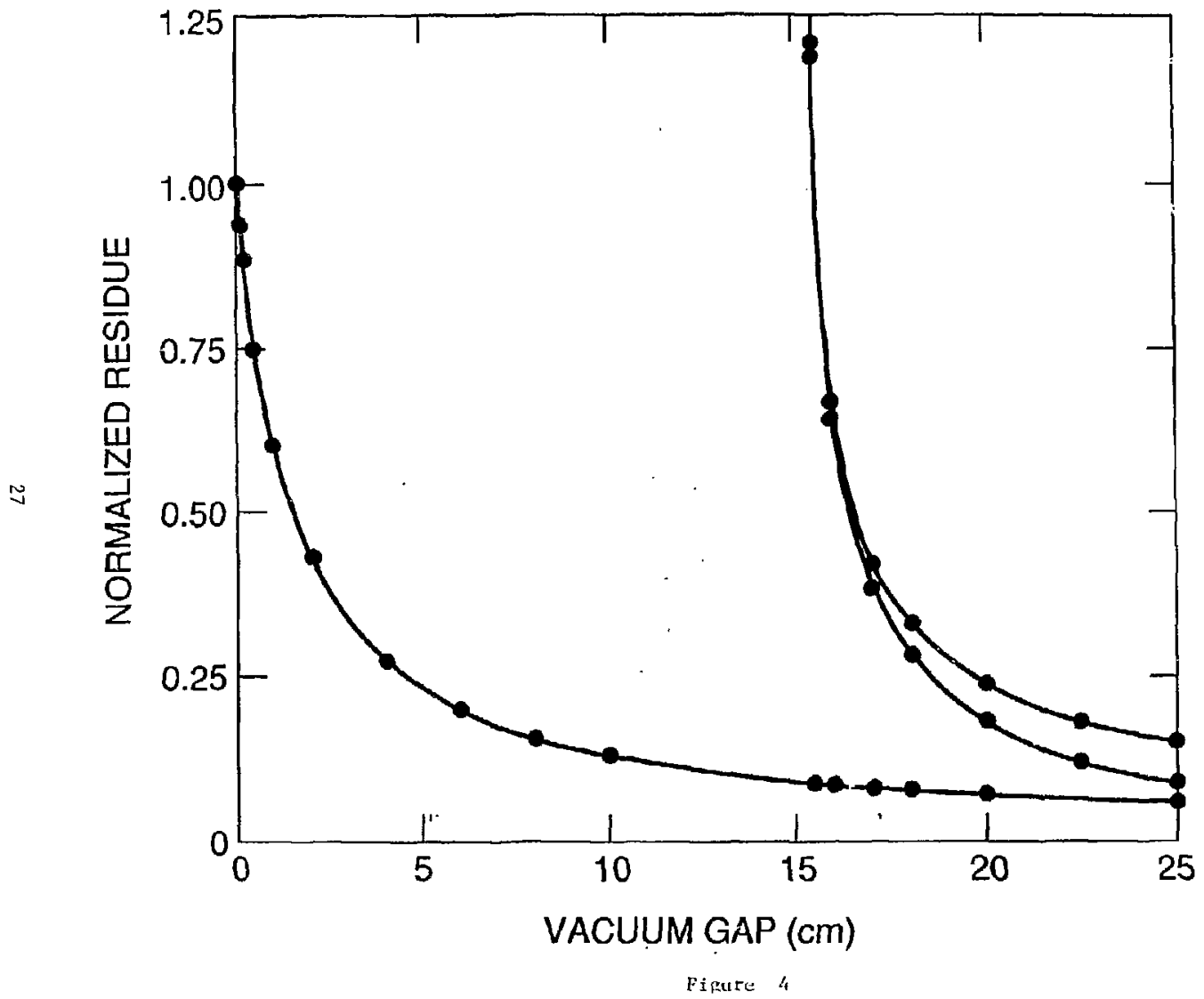




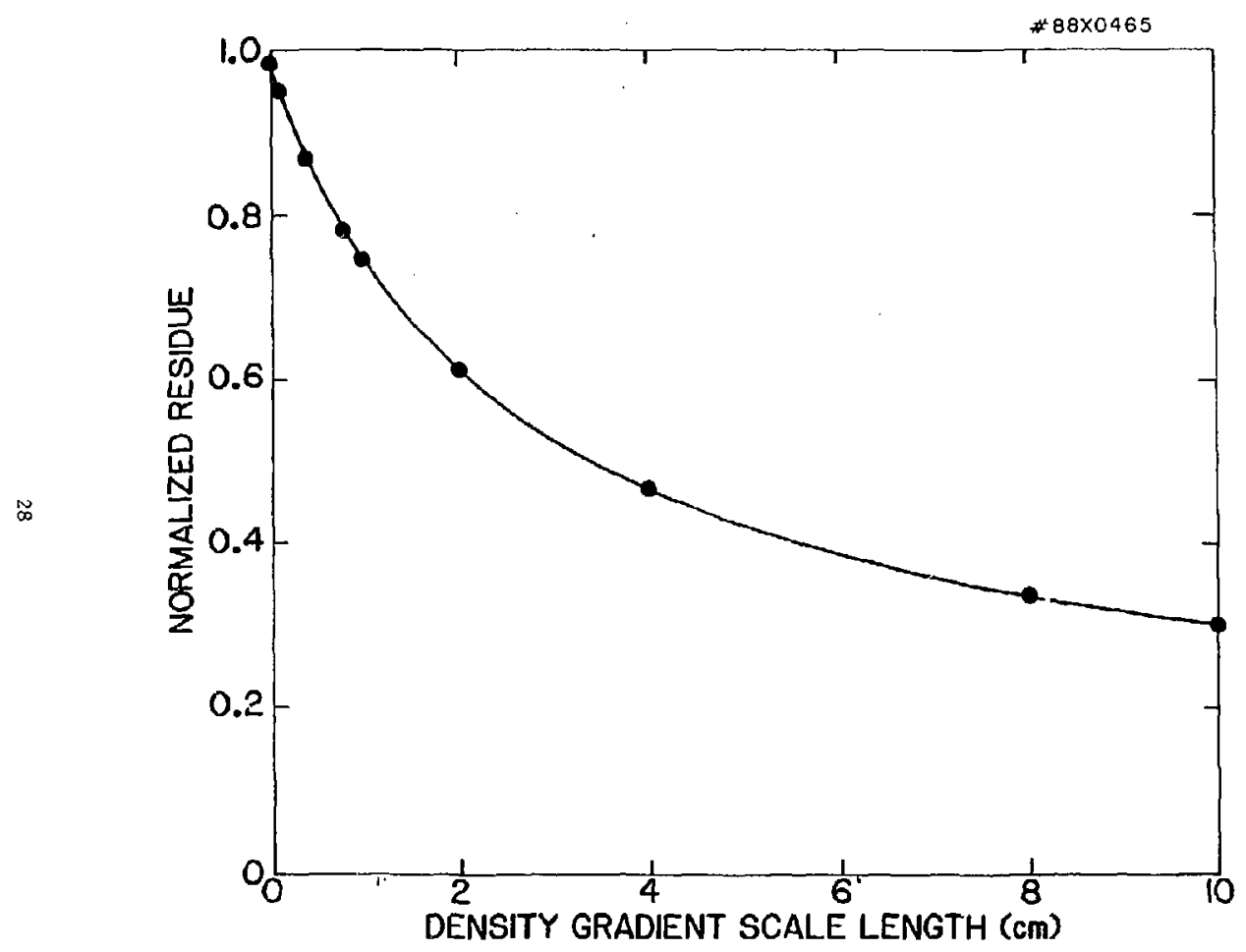

Nipure 5 

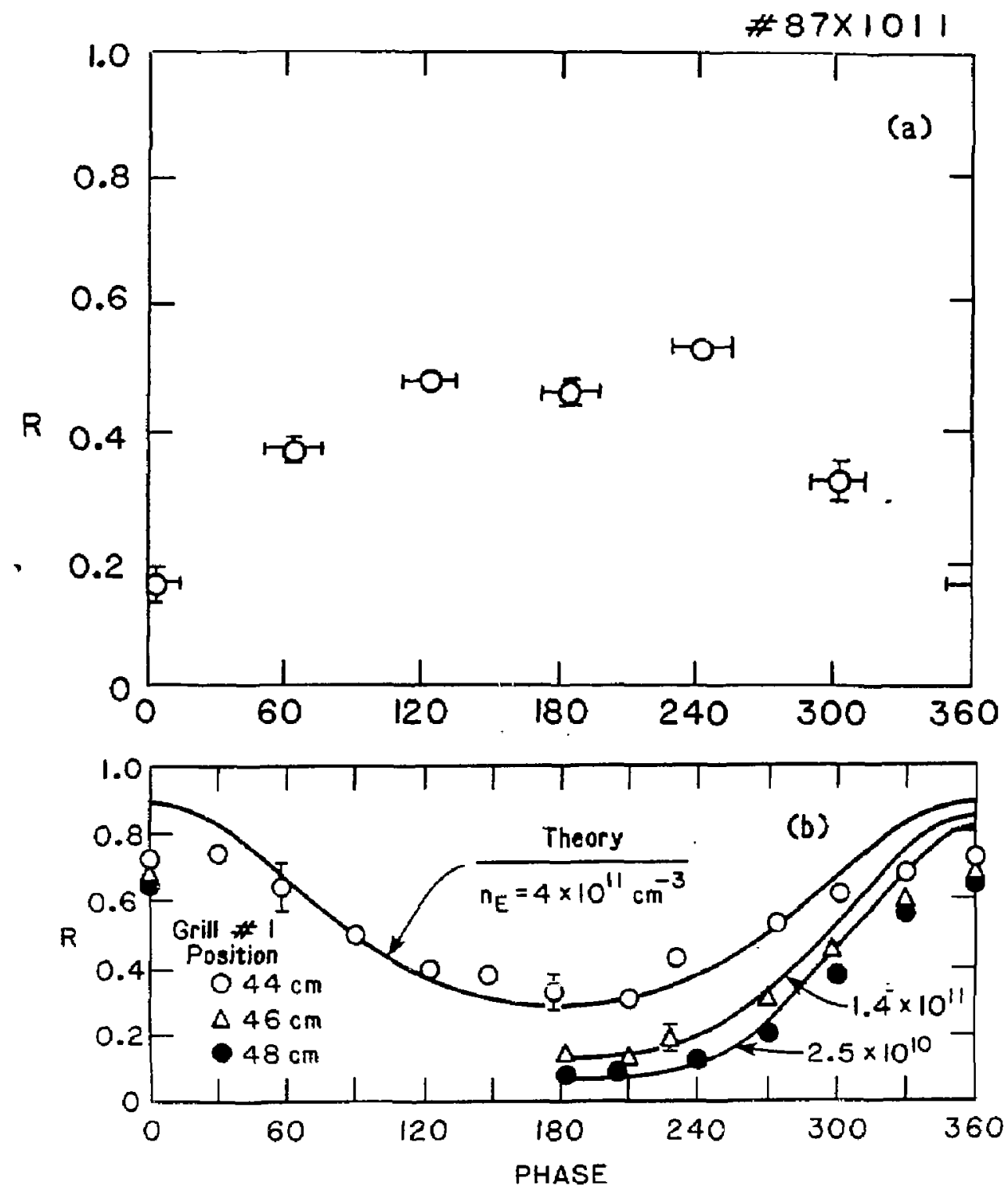

Figure 6 


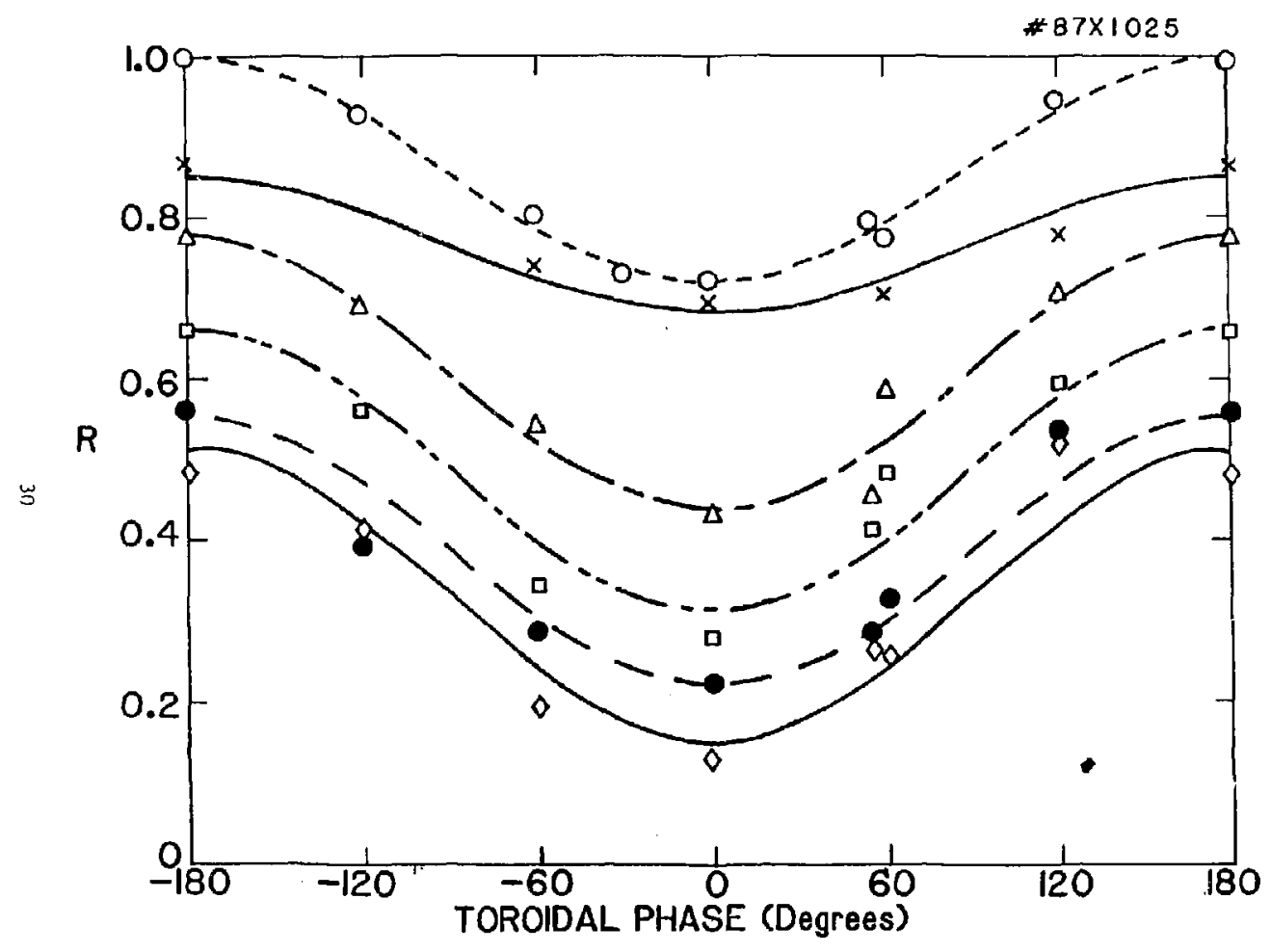

ligure 7 a 


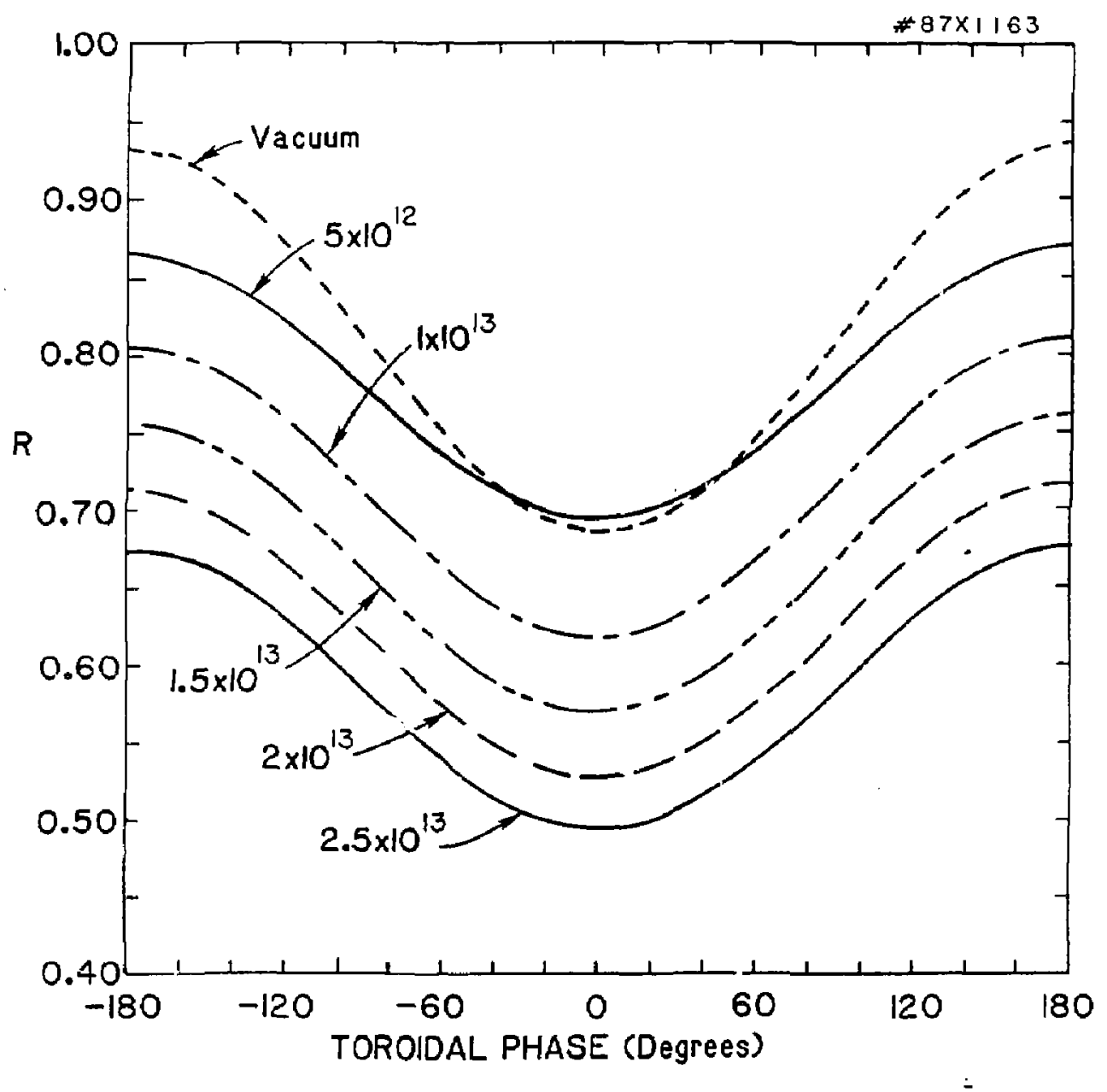

Figure 7b 


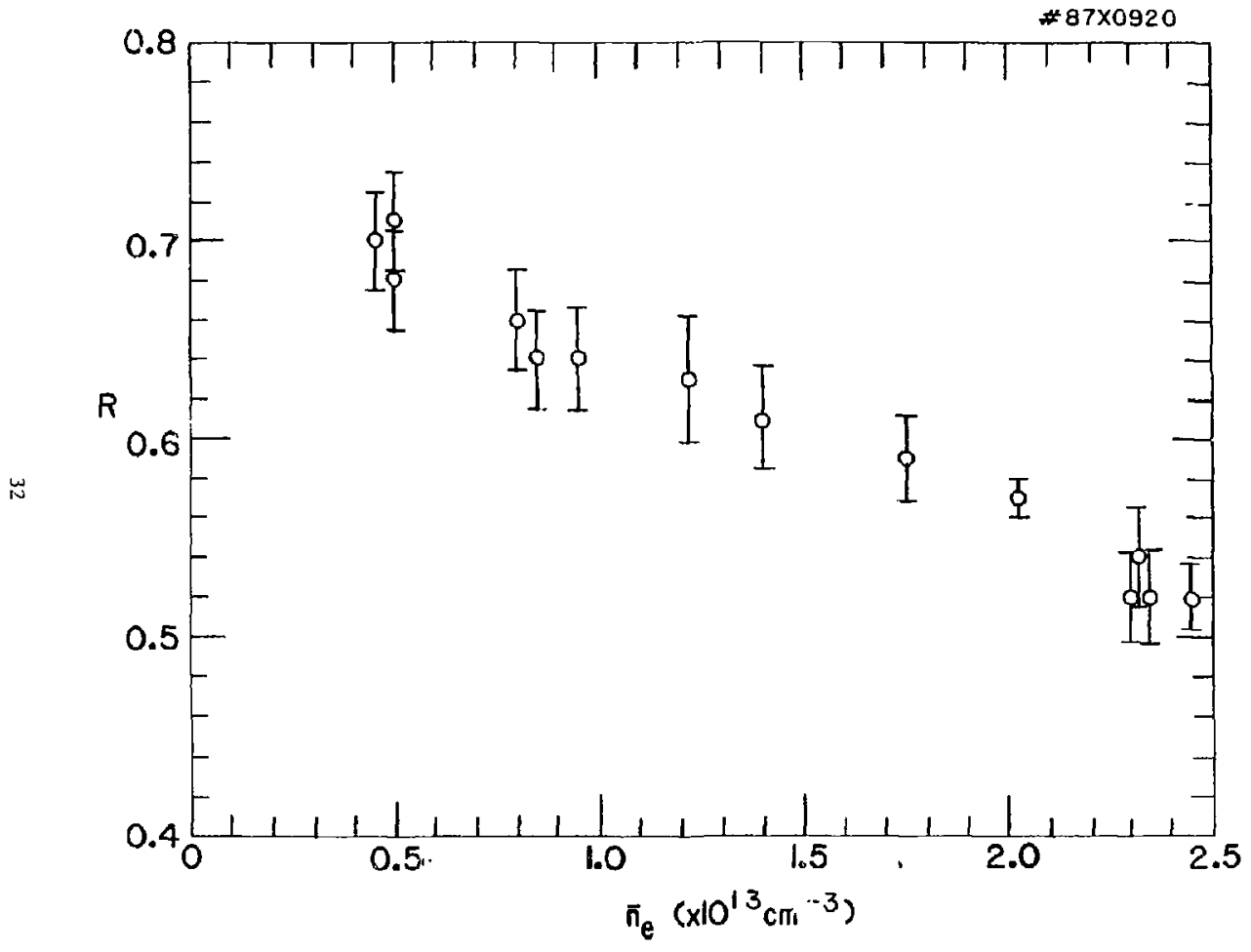

Tigure Ma 


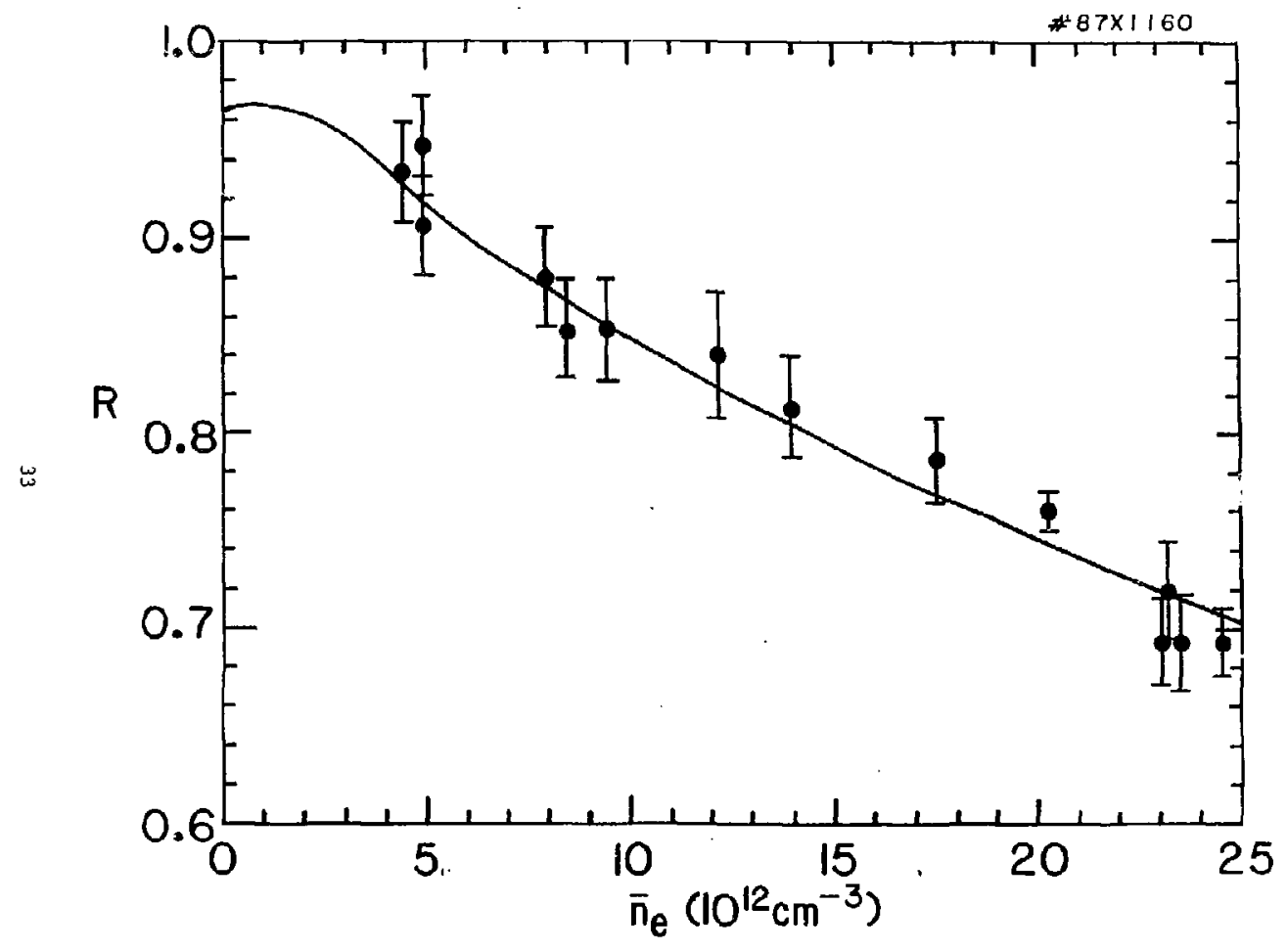

Figurt $8 h$ 


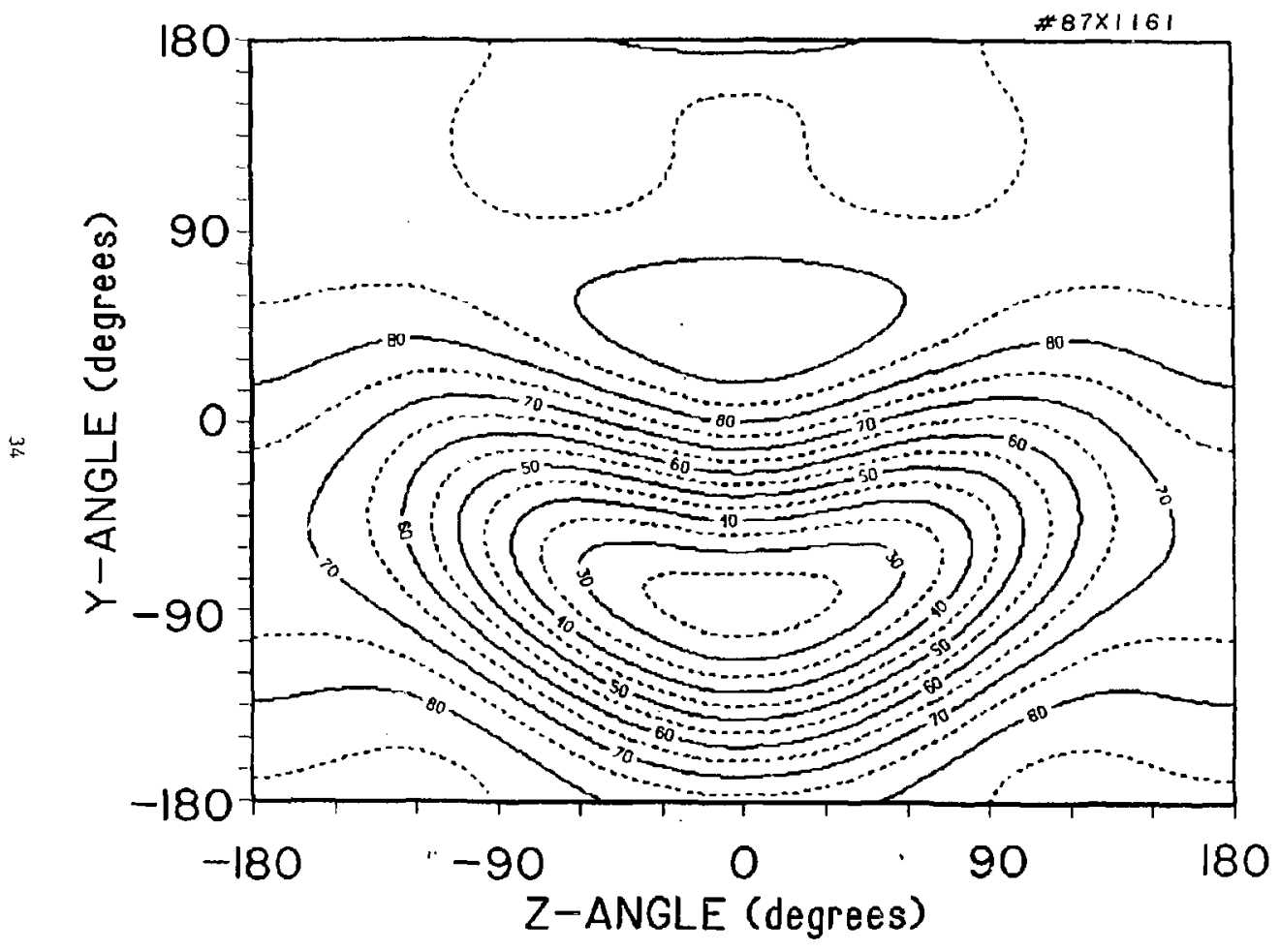

Figure 4 

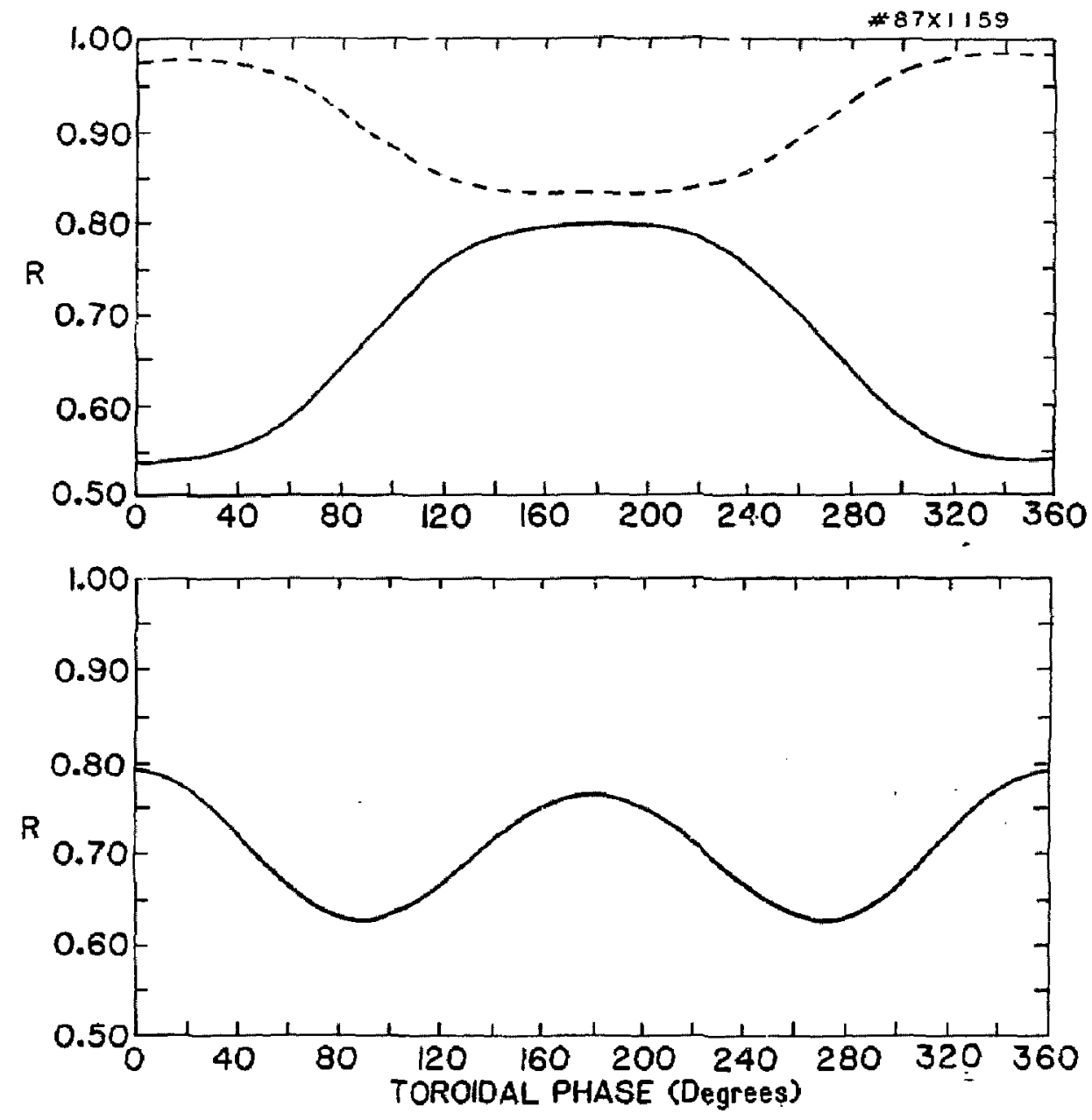

Figure 10 